\title{
The Efficacy of Mastoid Cavity Obliteration by Conchal Cartilage in Canal Wall Down Mastoidectomy
}

\author{
Al-Tayyar $\mathrm{M}^{*}$, Baban M, Khdhayer A, Najeeb R, and Othman S \\ Otolaryngology and head and neck surgery centre, Al-Sulaimaniah Teaching Hospital, Pasha City, Raperin Al-Sulaimaniah, \\ Iraq
}

*Corresponding author: Al-Tayyar M, Otolaryngology and head and neck surgery centre, Al-Sulaimaniah Teaching Hospital, Apartment 964, Pasha City, Raperin Al-Sulaimaniah, Iraq, Tel: +964 770604 5055, E-mail: dr.pilota@gmail.com

Citation: Al-Tayyar M, Baban M, Khdhayer A, Najeeb R, Othman S (2018) The Efficacy of Mastoid Cavity Obliteration by Conchal Cartilage in Canal Wall Down Mastoidectomy. J Otolaryngol Res 1: 103

Article history: Received: 30 March 2018, Accepted: 15 June 2018, Published: 18 June 2018

\section{Abstract}

Introduction: CWD (canal wall down) mastoidectomy is considered the best method to treat chronically discharging ear. This technique shows its efficacy in eradicating the disease process from the middle ear cavity. However, it contributes to the formation of a large cavity, which causes several problems. To get rid of these problems, mastoid obliteration is done.

Aim and objectives: To study the outcomes and sequences of mastoid obliteration with conchal cartilage.

Materials and methods: the study was conducted retrospectively on 30 patients who had ear cholesteatoma and underwent a CWD mastoidectomy with mastoid obliteration by the conchal cartilage, tympanic membrane reconstruction and ossiculoplasty by either partial or total ossicular replacement prosthesis.

Results: all the patients had complete epithelization and no disease recurrence. Retraction pockets were found in 3 patients (10\%) with debris and wax in 6 patients (20\%). 1 case had no problem in fitting her hearing aid and a patient had complete graft resorption. The meatuses were aesthetically accepted in all patients, as they were not too wide.

Conclusion: mastoid obliteration by conchal cartilage is a very effective method to prevent problems resulted in cavity formation after CWD mastoidectomy.

Keywords: Cholesteatoma; Conchal Cartilage; Mastoidectomy; Obliteration

\section{Introduction}

A chronic discharging ear has been considered a real problem and a challenge for the otologists for a long period. The main objective in the surgery of aural cholesteatoma is to completely eradicate the disease to obtain a safe and a dry ear. The other objective is to restore the hearing capacity of the ear as much as possible. The open mastoidectomy technique has been the mainstay of the management of chronic ear disease due to cholesteatoma; however, this type of surgery cannot be carried on without related consequences. It results in the formation of a mastoid cavity, which is unnatural and anatomically and physiologically unacceptable. The problems that result from an open cavity are as follows:

1- Chronic discharge from the open cavity with the incidence of discharge being $20-60 \%$.

2- Dependence on the surgeon for regular cleaning of the cavity.

3- Episodic vertigo due to exposure of semi-circular canals to the direct caloric stimulation by air and/or water entering the cavity.

4- Unsightly appearance due to a large meatoplasty.

5- Difficulty in placing hearing aids to people who need them $[1,2]$

On the other hand, canal wall up (CWU) technique doesn't need to disrupt the anatomical configuration of the middle ear cleft, but it is associated with a higher rate of incomplete eradication of the disease [3-5]. From above, it is clear that both CWU and CWD techniques have their own advantages and disadvantages. Hence, it was decided to find out whether it is possible to combine the advantages and, at the same time, circumvent the disadvantages of both the CWU and CWD techniques. The approach was 
mastoid and epitympanic obliteration followed by posterior bony canal wall reconstruction (CWR) and ossiculoplasty to eradicate the disease and at the same time avoid the problems of the open cavity [3]. The obliteration should also not affect the primary and secondary objectives of the aural surgery.

In a historical point of view, Mosher being the first to introduce this concept in 1911 as he produced the first literature about mastoid obliteration $[1,6]$. Since then, many authors described many surgical obliteration techniques using several materials (either synthetic or biological) to solve the mastoid cavity problems. One of these obliterating materials is the cartilage, which is used for the first time by Peer in 1943 to fill the mastoid defect in retroauricular fistulas [7,8].

\section{Material and Methods}

This study was performed as a retrospective review of the clinical database collected from 30 patients who underwent canal wall down mastoidectomy with the obliteration of the mastoid cavity by a conchal cartilage in Al-Sulaimaniah teaching hospital and Zhian private hospital in Al-Sulaimaniah city in Iraq. Patients who were included in this study underwent a canal wall down mastoidectomy for a cholesteatoma of the middle ear cleft. Meanwhile, any case with a canal wall up or canal wall down mastoidectomy for other reasons is excluded from this study. The data that fulfilled the above criteria in a period of 31 months from May 2014 to January 2017 was retrieved.

Among those thirty patients, twenty of them had their surgeries for the first time (primary cases), while the remaining ten patients had undergone a previous surgery in the involved ear (revision surgeries) who had previous mastoid cavities and discharging ears. All the patients had surgeries on one side. A database of the following parameters was collected, which include: patient's age, gender, pre-operative signs, symptoms, operated side, operative details and postoperative signs, symptoms, and complications. In addition to that, a pure tone audiometry (PTA) was done to evaluate air conduction (AC) and bone conduction (BC) with the calculation of the air-bone gap (ABG) pre- and postoperatively. A tympanogram was also done to all patients. All patients were followed-up on a postoperative day, 1 month, 3months, 6 months and more than 6 months. The patients were instructed to keep their operated ears dry in the immediate postoperative period and to return to the clinic if they noticed any evidence of wound infection (like pain and discharge).

\section{Surgical technique}

All the patients had their surgeries under general anesthesia. The operation began by injecting the external canal and the postauricular area with 2\% xylocaine and 1:200000 adrenaline. A canal incision was made just medial to the osteocartilaginous junction. A postauricular incision was made about $0.5-1 \mathrm{~cm}$ behind the sulcus, which was extended superiorly in order to harvest the temporalis fascia. The periosteum was incised as a T-shaped incision and elevated by periosteal elevator then connected to the canal incision. The tympano-meatal flap was raised. Then, CWD mastoidectomy was performed either by outside-in or inside-out technique according to the disease extension and the size of the mastoid antrum. The eroded ossicles were removed and the whole of the mastoid \& the middle ear cavity were cleared of the cholesteatoma. The conchal cartilage was obtained and cut into slices in order to obliterate the mastoid cavity and reconstruct the posterior and superior wall of the external auditory canal and it was covered by the temporalis fascia graft. The ossiculoplasty was done by either TORP or PORP according to the ossicular damage in almost all the patients (except one, who had a profound sensory neural hearing loss). Meatoplasty was performed at the end of the operation followed by packing and dressing. (Figure 1 and 2).

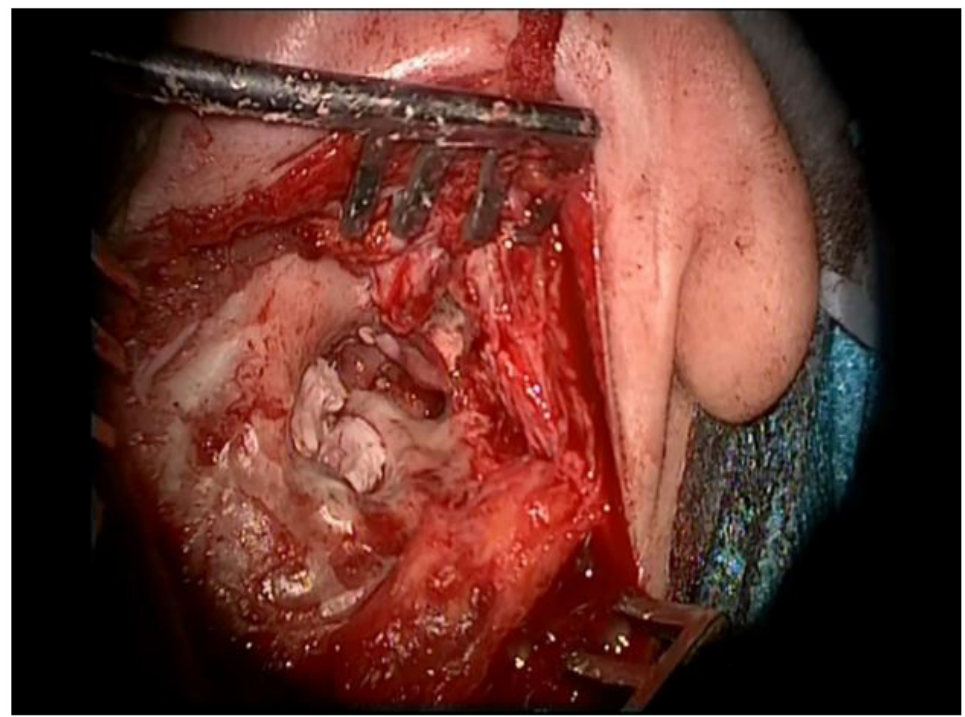

Figure 1: Conchal cartilage was cut into slices and put to fill the mastoid cavity and reconstruct the posterosuperior canal wall, intraoperative view 


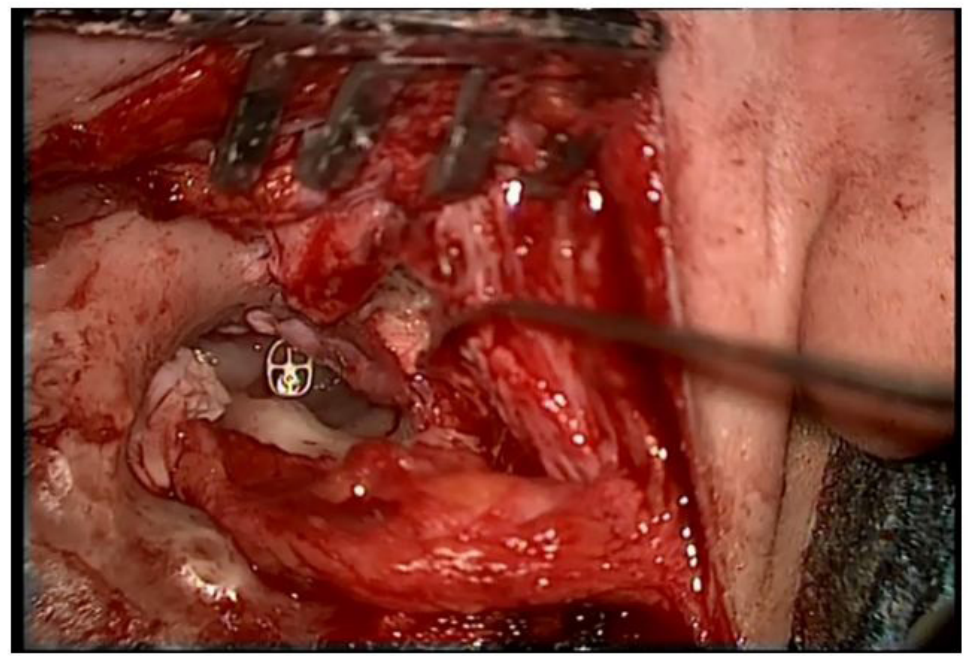

Figure 2: PORP insertion, intraoperative view

\section{Results}

Our study was conducted on 30 patients. Male patients were 13 patients (43.4\%), while the female patients were 17 (56.6\%). The age distribution of the patients ranged from 19 to 64 years (mean=39.6, standard deviation +12.9$)$. There was an equal distribution between the right and the left side that was operated. Every patient had undergone an operation on one side. 20 patients (66.7\%) had not operated before (primary surgery), while the remaining 10 patients (33.3\%) had been operated previously (revision surgery).

The presenting symptoms varied. Reduce hearing represented the majority of cases (29 patients, 97\%), followed by other presentations like discharging ear (25 patients, $83 \%$ ), tinnitus (15 patients, 50\%) and episodic vertigo or unsteadiness (10 patients, 33.3\%) (Table 1).

\begin{tabular}{|c|c|}
\hline Characteristic & Value \\
\hline $\begin{array}{c}\text { Age (year): mean, } \\
\text { range }\end{array}$ & $39.6(19-64)$ \\
\hline Gender & \\
\hline Male & $13(43.4 \%)$ \\
\hline Female & $17(56.6)$ \\
\hline Presenting symptoms & \\
\hline Reduce hearing & $29(97 \%)$ \\
\hline Discharge & $25(83 \%)$ \\
\hline Tinnitus & $15(50 \%)$ \\
\hline Vertigo & $10(33.3 \%)$ \\
\hline Surgery & \\
\hline Primary surgery & $20(66.7 \%)$ \\
\hline Revision surgery & $10(33.3 \%)$ \\
\hline
\end{tabular}

Table 1: Demographics of the patients

All the primary cases and a half of the revision ones were found to have a cholesteatoma in their diseased ears either pre- or intraoperatively, so they underwent a CWD mastoidectomy with mastoid obliteration by a conchal cartilage followed by ossicular reconstruction.

All patients showed complete epithelization with no disease recurrence within the follow-up period, which is performed by microscopic and endoscopic examination. Retraction pockets were found in 3 patients (10\%) in the reconstructed tympanic membranes parallel to the mesotympanum with debris and wax that were easily removed in 6 patients (20\%). The ABG mean was $42.2 \mathrm{~dB} \pm 10.8)$ pre-operatively, then, which became $27.4 \mathrm{~dB}( \pm 12.9)$ with $\mathrm{P}$ value $<0.05$.

Among those patients, 5 of them (16.7\%) showed abnormal epithelial migration which formed a "false membrane" over the reconstructed tympanic membrane after 6-12 months from their operations. That "false membrane" made the patients suffering from deterioration in their hearing despite their hearing improvement after their surgeries. That "false membrane" was removed under local anesthesia and debris were found in the space between those membranes and the reconstructed tympanic membranes. The patients' hearing was improved after that removal. 
The meatuses were aesthetically accepted in all patients, as they were not too wide (mean of the external canal volume $=1.3 \mathrm{ml}$ $\pm 0.7 \mathrm{ml}$ ), except in one patient who was interested as there was a complete resorption of the graft material, resulting in wide canal, exposed mastoid cavity with ear discharge (the same problems of CWD mastoidectomy without obliteration) (Figure 3 ).

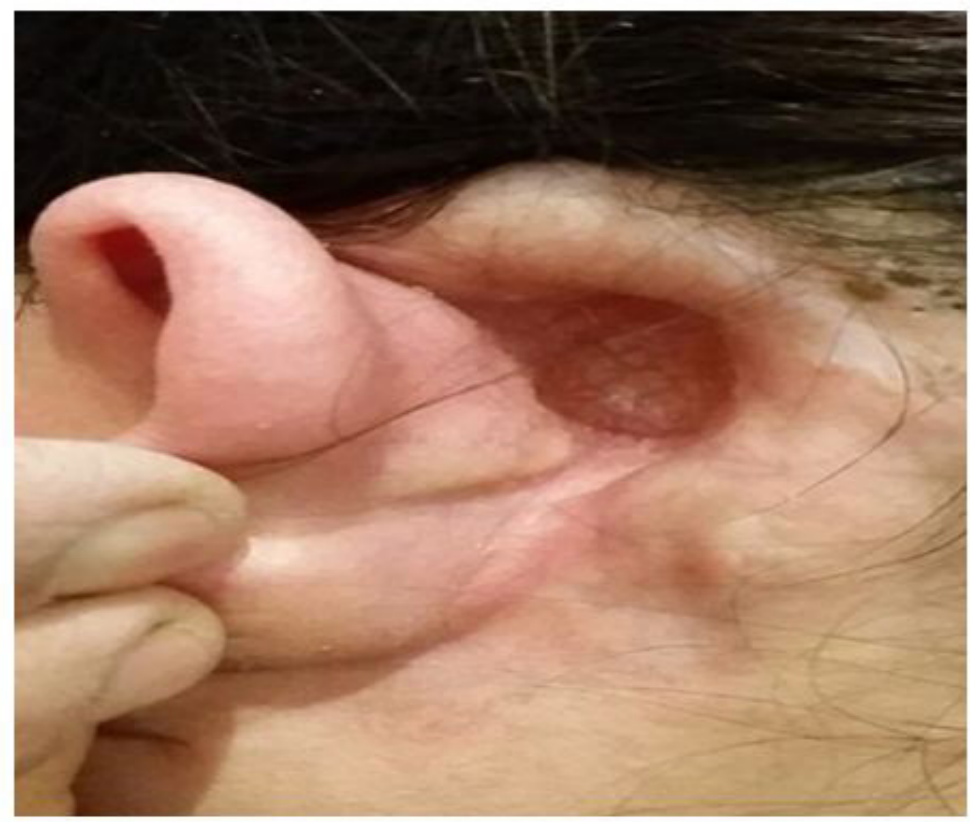

Figure 3: Rare complication. Complete resorption of the grafted cartilage resulted in an exposed mastoid cavity

\section{Discussion}

A common potential problem with traditional canal wall down (CWD) mastoidectomy procedures in treating cholesteatoma is the long-term morbidity associated with discharging cavities [3,9]. CWD is a destructive approach, as it creates an open cavity and alters the anatomy and physiology of the middle ear and the mastoid. This can result in some problems such as frequent ear discharge, difficulty in fitting a hearing aid, the need for water precautions to prevent caloric stimuli and a potential lifetime of visits to outpatients for aural care as the normal pathway of epithelial migration will be altered. This leads to a significant socioeconomic impact $[3,10]$.

It is worthy to mention that CWD mastoidectomy due to the removal of the cholesteatoma from the middle ear cleft is not the only indication for mastoid cavity obliteration. Other indications are: chronic otorrhea or nonhealing of the mastoid bowl, CSF leak, extensive temporal bone trauma, temporal bone resection for malignancy, cochlear implantation in patients with chronic otitis media, labyrinthitis ossificans in whom a drill out of cochlea is required with the removal of posterior canal wall and tympanic membrane, treatment of chronic otitis media in ears with no useful hearing $[1,11]$.

Another point to be mentioned as an advantage of the mastoid obliteration is its effect on the negative pressure in the middle ear and the mastoid, which contributes to the recurrence of cholesteatoma. Inflammatory conditions from a wide surface area of the mastoid cavity will result in more negative middle ear pressures as the diseased mucosa absorbs more gas $[3,12,13]$. Eradication of the diseases mastoid epithelium, followed by obliteration, can minimize these potential problems by reducing the size of the mastoid cavity and promoting epithelialization over the exposed mastoid bone $[2,14,15]$.

The obliterating material differs. It could be autologous, homologous or synthetic. The autologous material is considered the preferred one because of its availability during the operation and no worries are taken regarding the transmitted diseases or allergic rejection. The autologous materials include several items such as bone chips, bone pâté, tragal cartilage and conchal cartilage. In our study, we use conchal cartilage as an obliterating material.

In 1943, Peer used the first-time cartilage as a material to fill the mastoid defect in retro-auricular fistulas [7,8]. Although a cartilage graft tends to reduce consistency over time, it retains the shape, unlike bone, which tends to resorb [7,16]. This was one reason to choose the cartilage in obliteration. Cartilage fragmentations into smaller slices and their placement in mastoid cavities make the fibrous tissue grow in between healing tissue to fill the remaining gaps, thus effectively increasing the obliteration volume [7]. That was the other reason for choosing the cartilage.

\section{Conclusion}

Mastoid obliteration by conchal cartilage is a very effective method to prevent problems resulted in cavity formation after CWD 
mastoidectomy. A comparative study is better to be held between obliterating the mastoid cavity by conchal cartilage and other material (like bone chips) to find out the best material which can be used in obliteration.

\section{References}

1. Shraddha Deshmukh, Arpit Sharma, Jyoti Dabholkar (2012) Mastoid cavity obliteration: Our experience. Otolaryngologia Polska 66: 379-81.

2. Males AG, Gray RF (1991) Mastoid surgery: quantifying the distress in a radical cavity. Clin Otolaryngol 19: 194-8.

3. Shao-Cheng Liu, Chih-Hung Wang, Bor-Rong Huang (2015) Obliteration of Radical Cavities and total reconstruction procedure without staging after canal wall Down mastoidectomy: long-term results. Clin Exp Otorhinolaryngol 8: 230-6.

4. Gyo K, Sasaki Y, Hinohira Y, Yanagihara N (1996) Residue of middle ear cholesteatoma after intact canal wall tympanoplasty: surgical find $\neg$ ings at one year. Ann Otol Rhinol Laryngol 105: 615-9.

5. Wilson KF, Hoggan RN, Shelton C (2013) Tympanoplasty with intact canal wall mastoidectomy for cholesteatoma: long-term surgical outcomes. Otolaryngol Head Neck Surg. 149: 292-5.

6. Ojala K, Palva A (1982) Results of obliterative cholesteatoma surgery. Arch Otolaryngol 108: 1-3.

7. A. Maniu M. Cosgarea (2012) mastoid obliteration with concha cartilage graft and temporal muscle fascia. ORL J Otorhinolaryngol Relat Spec 74: 141-5.

8. Peer LA (1943) Diced cartilage grafts. Arch Otolaryngol 38: 156-65.

9. Singh V, Atlas M (2007) Obliteration of the persistently discharging mastoid cavity using the middle temporal artery flap. Otolaryngol Head Neck Surg 137: 433-8.

10. Gantz BJ, Wilkinson EP, Hansen MR (2005) Canal wall reconstruction tym $\neg$ panomastoidectomy with mastoid obliteration. Laryngoscope 115: 1734-40.

11. Mehta RP, Harris JP (2006) Mastoid Obliteration. Otolaryngol Clin North Am 39: 1129-42.

12. Edfeldt L, StrOmbAck K, Kinnefors A, Rask-Andersen H (2013) Surgical treatment of adult cholesteatoma: long-term follow-up using total re $\neg$ construction procedure without staging. Acta Otolaryngol 133: 28-34.

13. Roberson JB Jr, Mason TP, Stidham KR (2003) Mastoid obliteration: autogenous cranial bone pAte reconstruction. Otol Neurotol 24: 132-40.

14. Mahendran S, Yung MW (2004) Mastoid obliteration with hydroxyapatite cement: the Ipswich experience. Otol Neurotol 25: 19-21.

15. Ramsey MJ, Merchant SN, McKenna MJ (2004) Postauricular periosteal-pericranial flap for mastoid obliteration and canal wall down tympa $\neg$ nomastoidectomy. Otol Neurotol 25: 873-8.

16. Zahnert T, Huttenbrink KB, Murbe D, Bornitz M (2000) Experimental investigations of the use of cartilage in tympanic membrane reconstruction. Am J Otol 21: $322-8$. 